\title{
Microwaves Make Hydroformylation a Rapid and Easy Process
}

\section{Elena Petricci, André Mann, ${ }^{\star}$ Angèle Schoenfelder, ${ }^{\star}$ Andrea Rota ${ }^{\#}$ and Maurizio Taddei*}

Dipartimento Farmaco Chimico Tecnologico, Università degli Studi di Siena, Via A. Moro, 53100 Siena, Italy.

CEM Italia srl, Via dell Artigianato, 6/8 24055 Cologno al Serio (BG), Italy

Laboratoire de Pharmacochimie de la Communication Cellulaire, CNRS-UMR7175, 74, rue du Rhin, BP 60024, F-67401 Illkirch, France

taddei.m@unisi.it

Supplementary Material.

General experimental procedure

pag 2

Microwave plot

pag 3

Characterisation of $\mathbf{2}$

pag 4

Characterisation of $\mathbf{4 a}$

pag 5

Characterisation of $\mathbf{6 a}$

pag 6

Characterisation of $\mathbf{8}$

pag 7

Characterisation of $\mathbf{1 0}$

pag 8

Characterisation of $\mathbf{1 2}$

pag 9

Characterisation of $\mathbf{1 4}$

pag 10

Characterisation of $\mathbf{1 6}$

pag 12

Characterisation of $\mathbf{1 9}$

pag 13

Characterisation of $\mathbf{2 2}$

pag 15

Characterisation of $\mathbf{2 5}$

pag 17

Characterisation of $\mathbf{2 6}$

pag 19

${ }^{1} \mathrm{H}$ NMR spectrum of the mixture of $\mathbf{2 4}$ and $\mathbf{2 7}$

pag 21

Characterisation of $\mathbf{2 0}$

pag 22 
General experimental conditions:

In a typical experiment the starting alkene $(0.64 \mathrm{mmol})$ was dissolved in toluene $(4 \mathrm{~mL})$. $\left(\mathrm{Ph}_{3} \mathrm{P}\right)_{3} \mathrm{Rh}(\mathrm{CO}) \mathrm{H}(0.013 \mathrm{mmol})$, XANTPHOS $(0.05 \mathrm{mmol})$ and $[\mathrm{bmim}]\left[\mathrm{BF}_{4}\right](187 \mu \mathrm{L})$ were added. The yellow solution obtained was submitted to pressurized syngas at 40 psi (2.7 Atm) and heated for 4 minutes at $110^{\circ} \mathrm{C}$ by microwave irradiation at $150 \mathrm{~W}$ (value previously settled on the Microwave oven. The flask was cooled and the internal gas relaesed. $\mathrm{Et}_{2} \mathrm{O}(10 \mathrm{~mL})$ was added to the reaction mixture and washed twice with $\mathrm{H}_{2} \mathrm{O}(5 \mathrm{~mL} \times 2)$; the organic layers were washed with brine $(5 \mathrm{~mL})$ and dried on dry $\mathrm{Na}_{2} \mathrm{SO}_{4}$. After filtration and evaporation in vacuo the yellow oil obtained was purified by flash chromatography. 
Microwave report:

Sample ID Null

Method el1 08/03/2006 12.00.10

$\begin{array}{lccccccc}\text { Stage } & 1 & 2 & 3 & & 4 & \\ \text { Power } & 150 & 0 & 0 & 0 & 0 \\ \text { Ramp Time } & 120 & 0 & 0 & 0 & 0 \\ \text { Hold Time } & 120 & 0 & 0 & 0 & 0 \\ \text { Temperature } & 110 & 0 & 0 & 0 & 0 \\ \text { Pressure } & 250 & 0 & 0 & 0 & 0 \\ \text { Stirring } & \text { True } & \text { True } & \text { True } & \text { True } & \text { True } \\ \text { Cooling } & \text { False } & \text { False } & \text { False } & \text { False } & \text { False }\end{array}$

Pressure and temperature window.

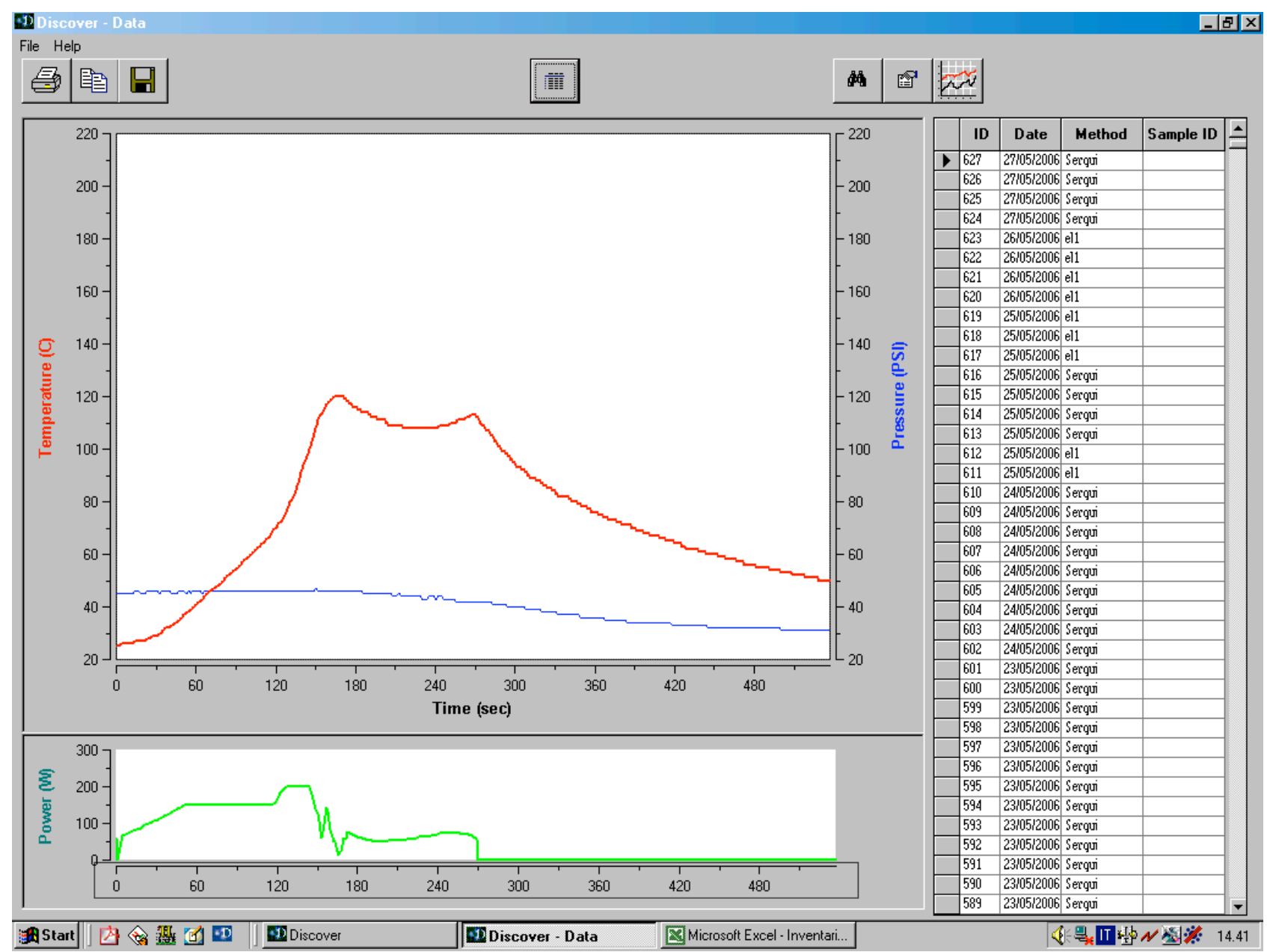




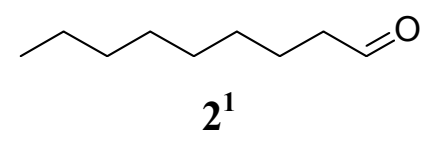

${ }^{1} \mathrm{H}$ NMR (200 MHz,CDCl$\left.{ }_{3}\right) 0.85(\mathrm{t}, J=6.6,3 \mathrm{H}), 1.20(\mathrm{~m}, 10 \mathrm{H}), 1.59(\mathrm{~m}, 2 \mathrm{H}), 2.41(\mathrm{~m}, 2 \mathrm{H}), 9.73$ $(\mathrm{s}, 1 \mathrm{H})$.

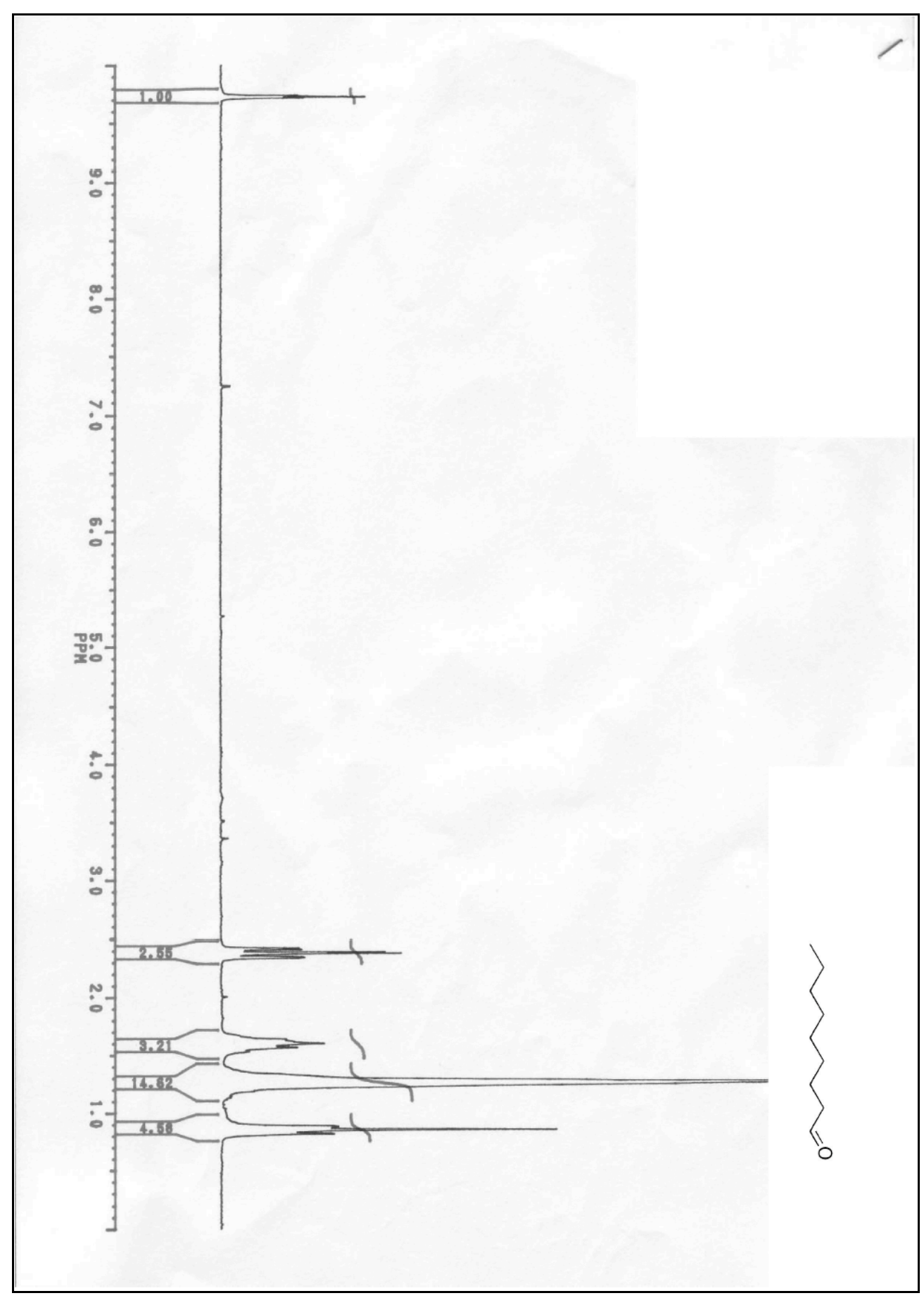




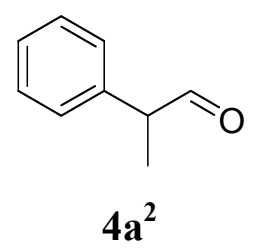

${ }^{1} \mathrm{H}$ NMR (200 MHz,CDCl $) 1.42(\mathrm{~d}, J=7.0,3 \mathrm{H}), 3.61(\mathrm{q}, J=7.0,1 \mathrm{H}), 7.21(\mathrm{~m}, 3 \mathrm{H}), 7.33(\mathrm{~m}$, 2H), $9.67(\mathrm{~s}, 1 \mathrm{H})$.

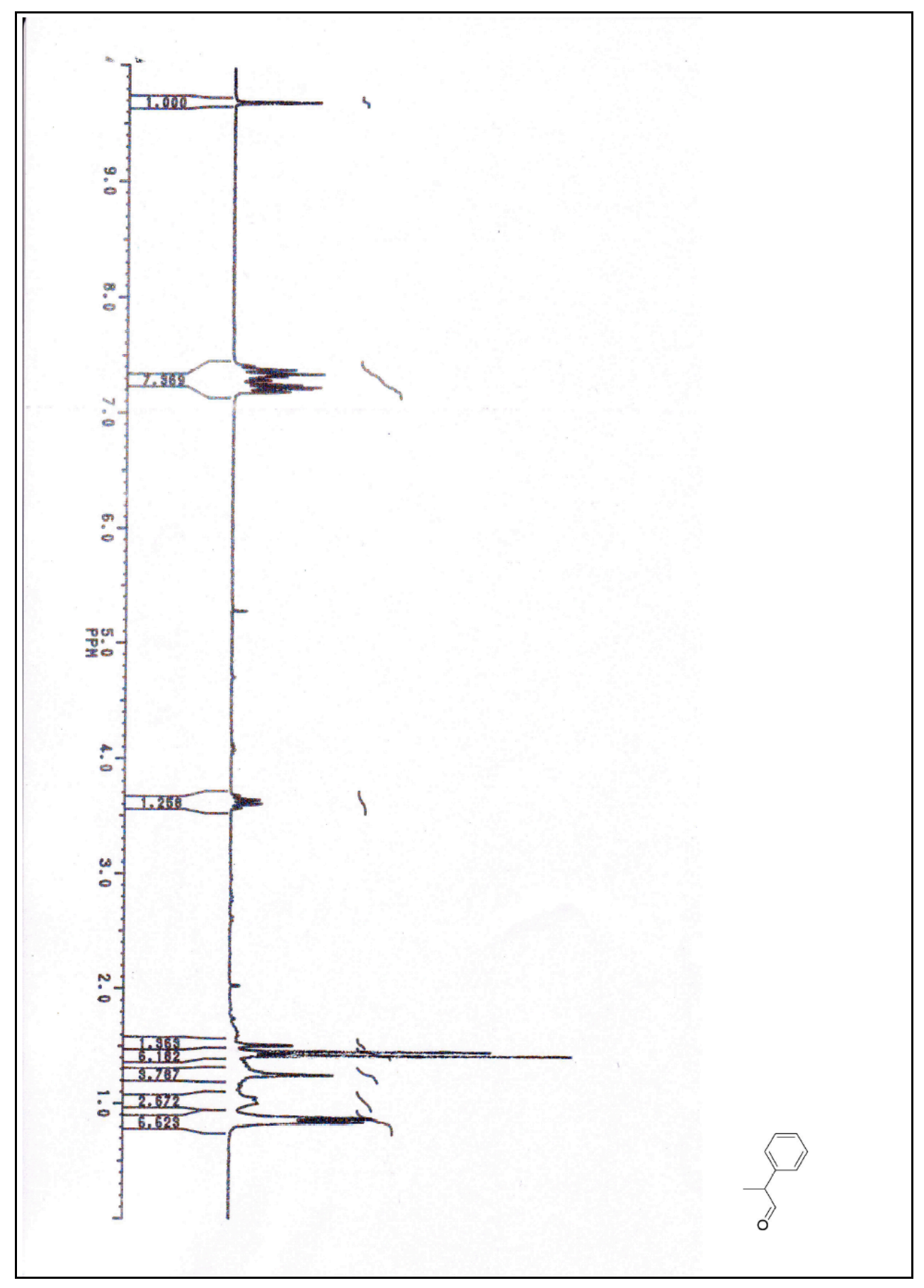




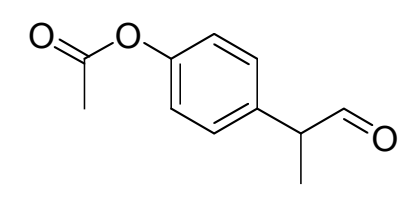

$6 \mathbf{a}^{3}$

${ }^{1} \mathrm{H}$ NMR (200 MHz, $\left.\mathrm{CDCl}_{3}\right) 1.46(\mathrm{~d}, J=7.2,3 \mathrm{H}), 2.34(\mathrm{~s}, 3 \mathrm{H}), 3.56(\mathrm{q}, J=7.2,1 \mathrm{H}), 7.15(\mathrm{~d}, J=$ $6.8,2 \mathrm{H}), 7.26(\mathrm{~d}, J=6.8,2 \mathrm{H}), 9.68(\mathrm{~s}, 1 \mathrm{H})$.

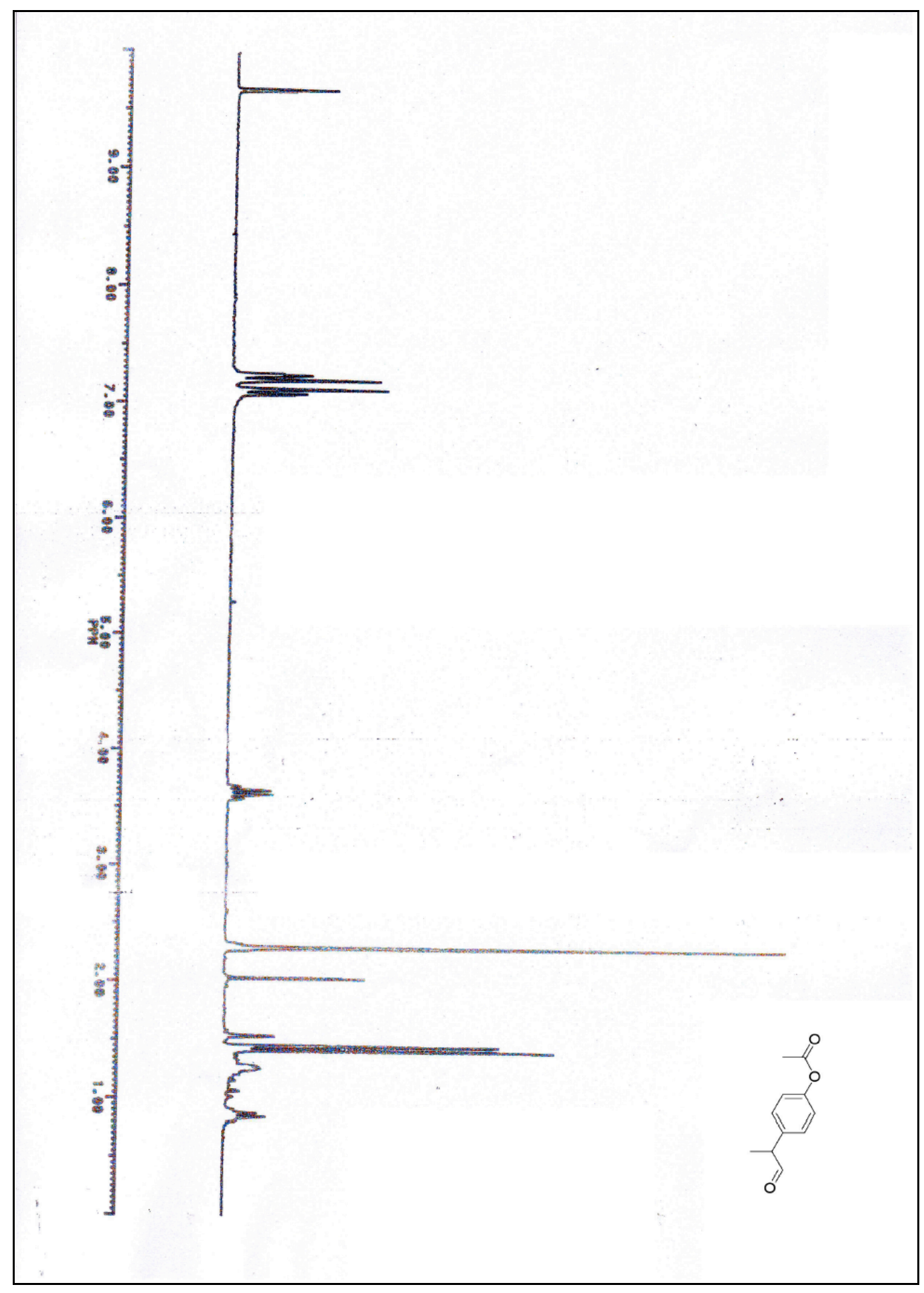




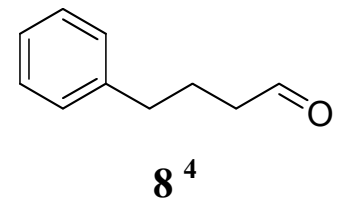

${ }^{1} \mathrm{H}$ NMR $\left(200 \mathrm{MHz}, \mathrm{CDCl}_{3}\right) 1.95(\mathrm{~m}, 2 \mathrm{H}), 2.45(\mathrm{t}, J=7.2,2 \mathrm{H}), 2.65(\mathrm{t}, J=7.2,2 \mathrm{H}), 7.25(\mathrm{~m}, 5 \mathrm{H})$, $9.86(\mathrm{~s}, 1 \mathrm{H})$.
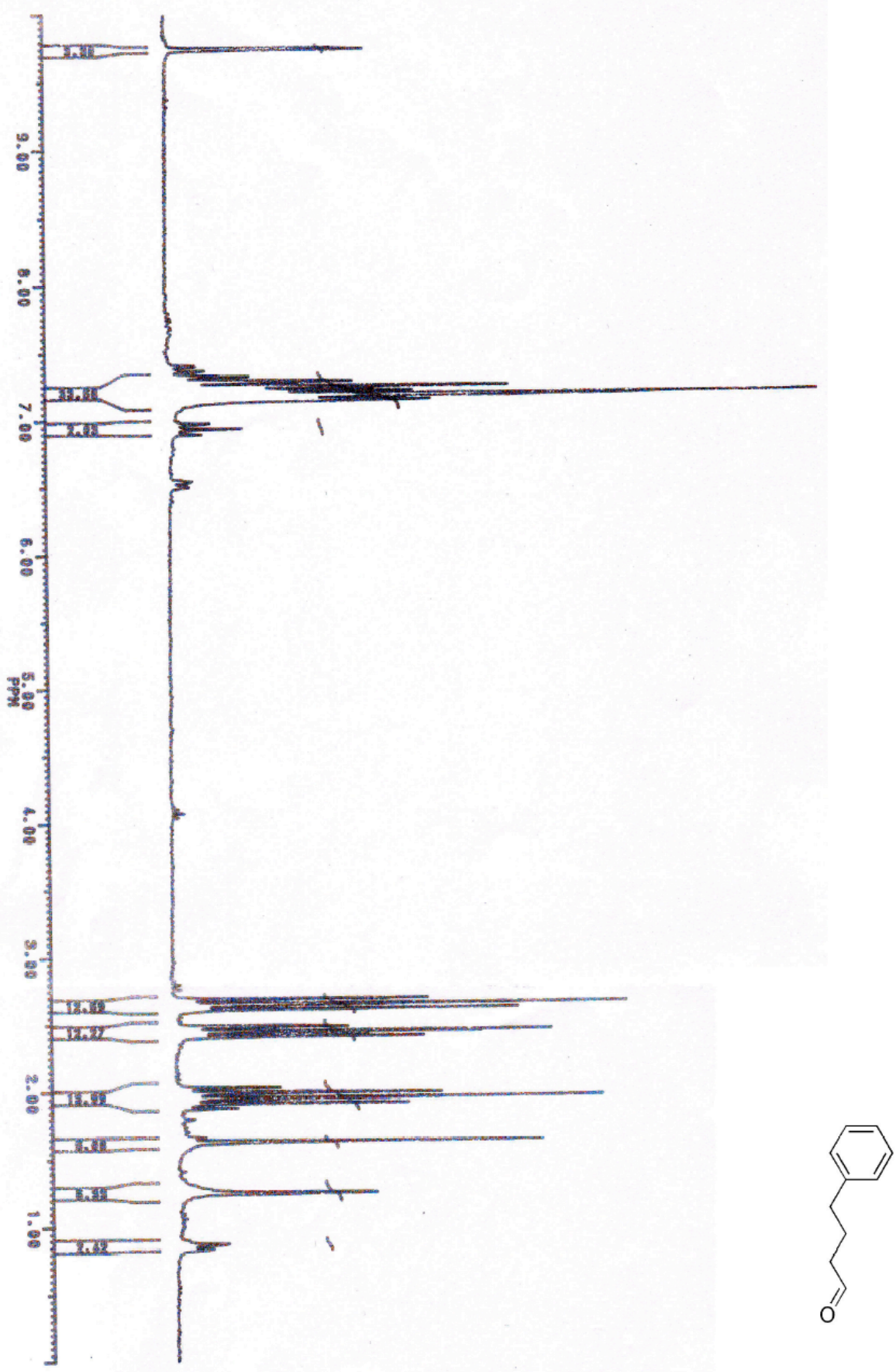
<smiles>CCOC(=O)CCCCCC=O</smiles>

${ }^{1} \mathrm{H} \mathrm{NMR}\left(200 \mathrm{MHz}, \mathrm{CDCl}_{3}\right) 1.16(\mathrm{t}, J=7.1,3 \mathrm{H}), 1.58(\mathrm{~m}, 4 \mathrm{H}), 2.21(\mathrm{~m}, 2 \mathrm{H}), 2.34(\mathrm{~m}, 2 \mathrm{H}), 4.03$ (q, $J=7.1,2 \mathrm{H}) 9.67(\mathrm{~s}, 1 \mathrm{H})$.

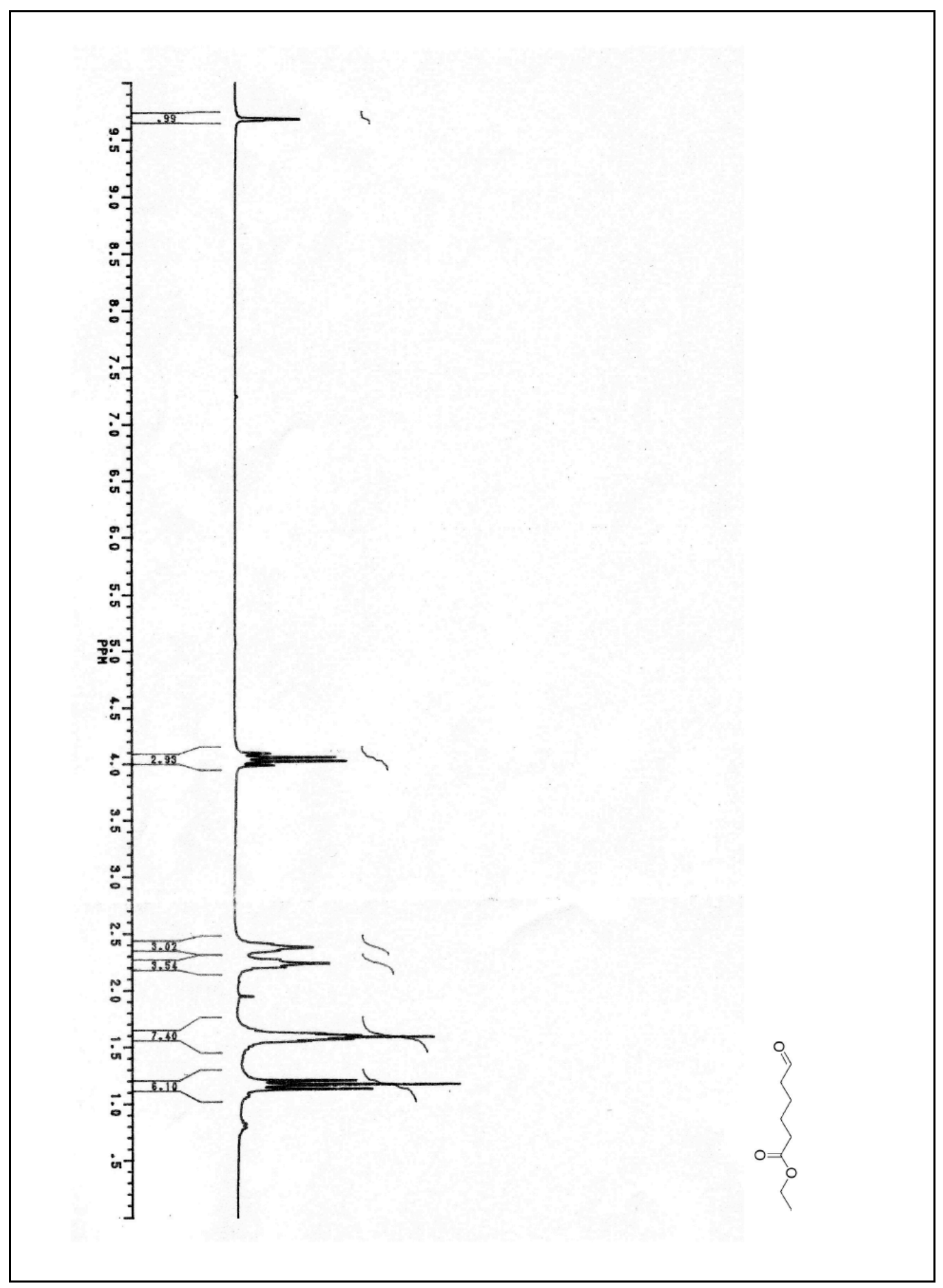

8 


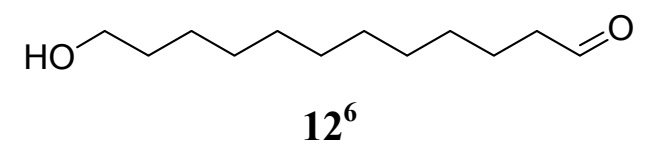

${ }^{1} \mathrm{H}$ NMR (200 MHz,CDCl $) 1.33(\mathrm{~m}, 12 \mathrm{H}), 1.55(\mathrm{~m}, 4 \mathrm{H}) 1.82(\mathrm{~m}, 2 \mathrm{H}), 2.39(\mathrm{t}, 2 \mathrm{H}), 3.62$, (t, 2H), ), $9.74(\mathrm{~s}, 1 \mathrm{H})$.

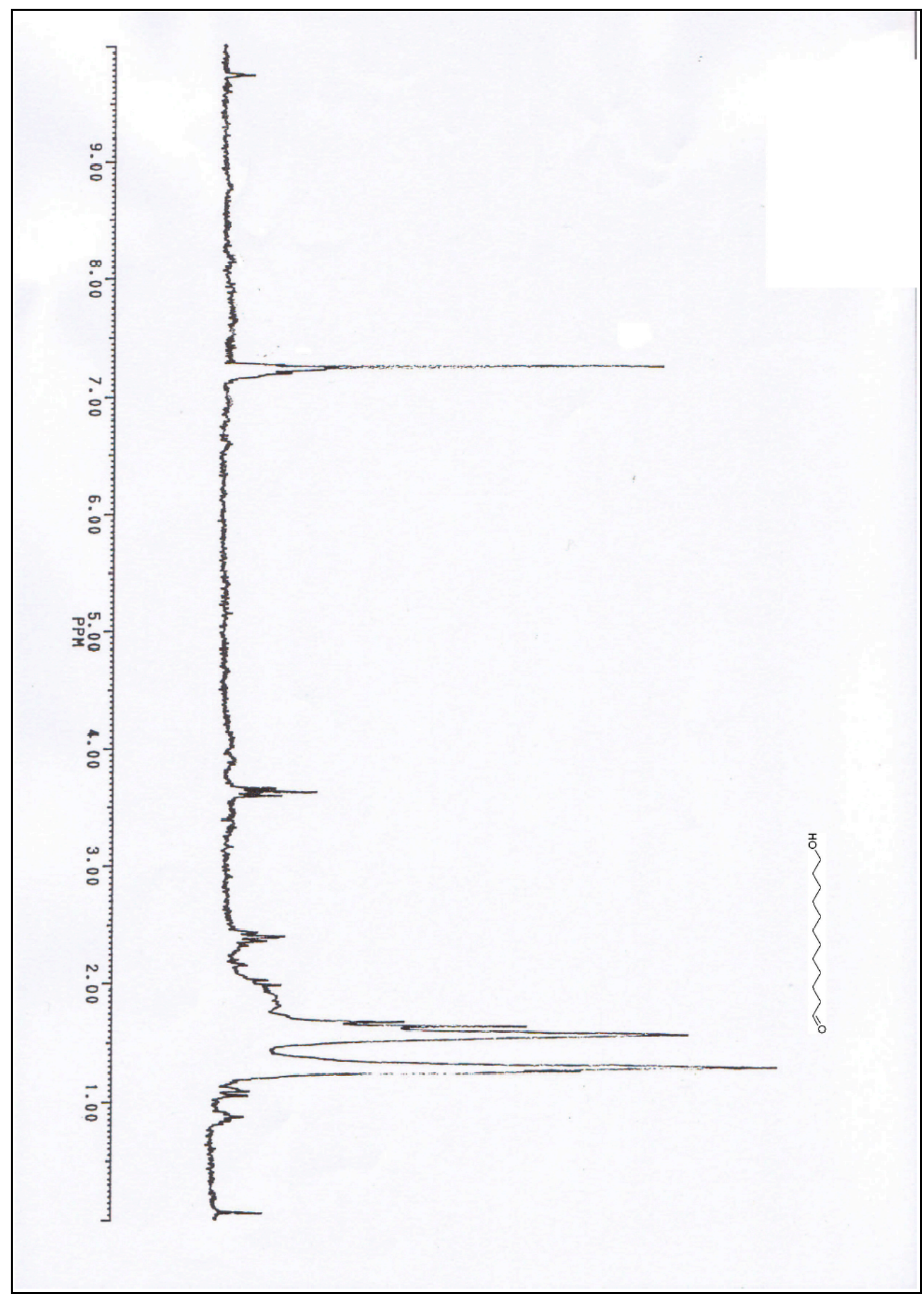




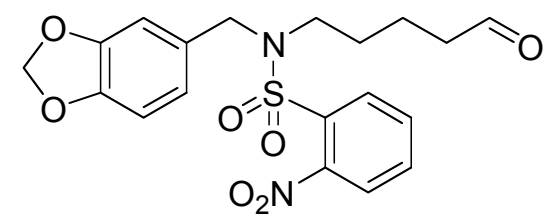

14

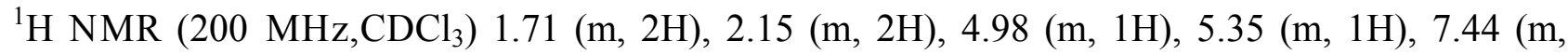
11H). ${ }^{13} \mathrm{C}$ NMR (50 MHz, $\mathrm{CDCl}_{3}$ ) 29.68, 39.40, 55.41, 195.10, 123.02, 125.49, 126.83, 128.39, 137.88, 152.99. $\mathrm{m} / z$ : ES/MS $302[\mathrm{M}+\mathrm{Na}]^{+}$. HRMS Calcd for $\mathrm{C}_{19} \mathrm{H}_{20} \mathrm{~N}_{2} \mathrm{O}_{7} \mathrm{~S}$, 420.0991. Found 420.0989 .
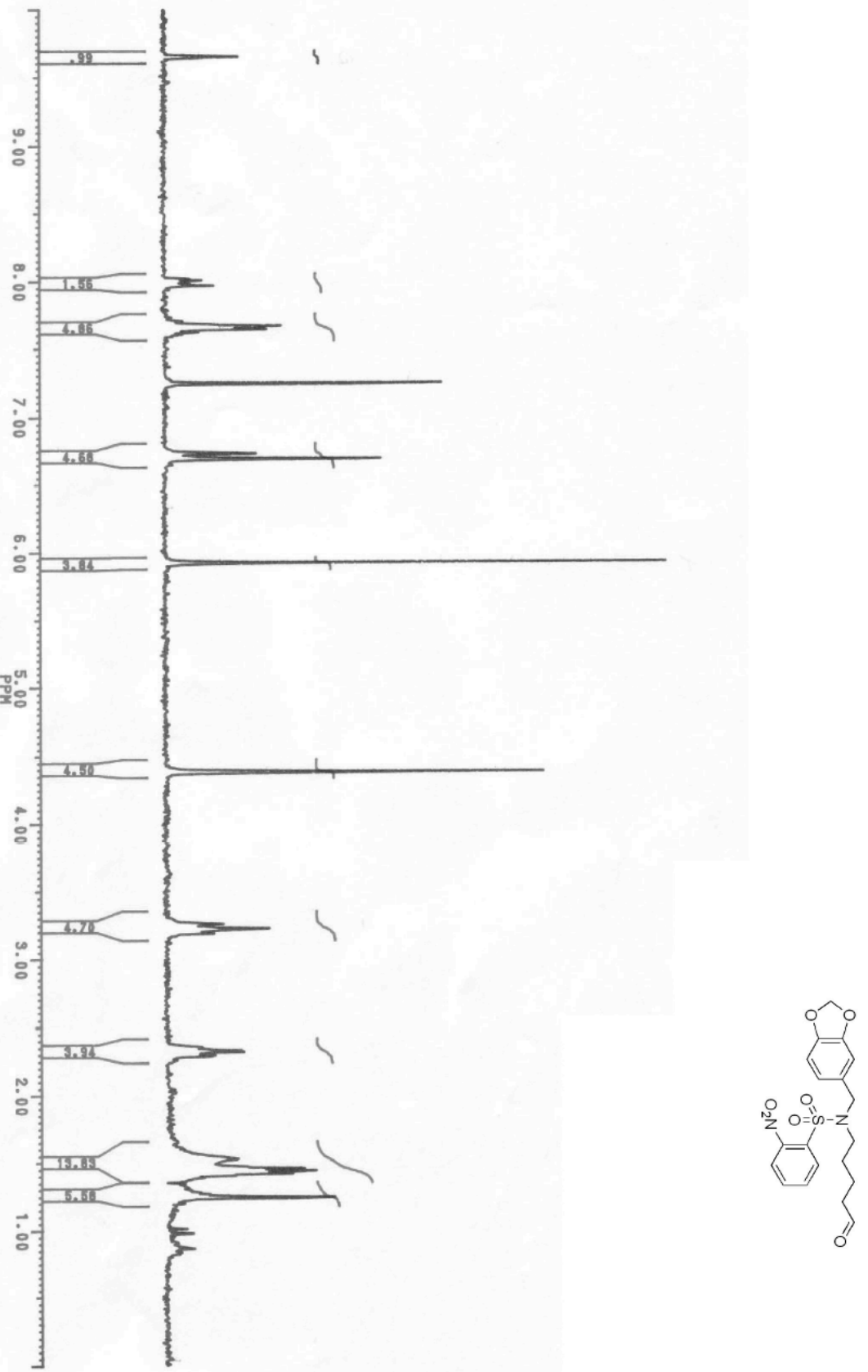


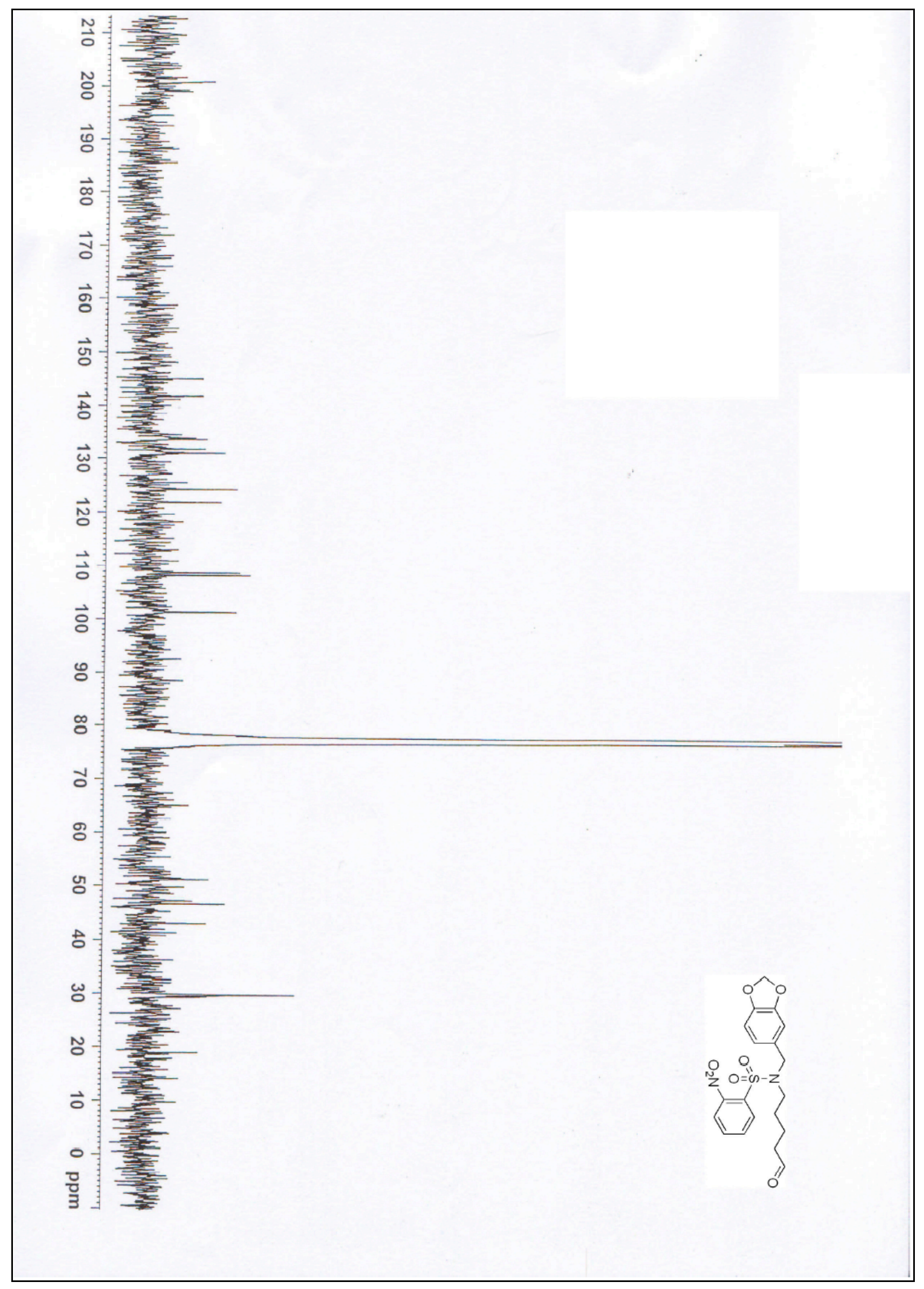




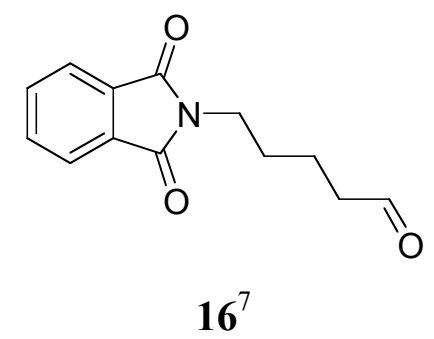

${ }^{1} \mathrm{H}$ NMR (200 MHz,CDCl 3 ) $1.75(\mathrm{~m}, 4 \mathrm{H}), 2.52(\mathrm{~m}, 2 \mathrm{H}), 3.78(\mathrm{~m}, 2 \mathrm{H}), 7.71(\mathrm{~m}, 2 \mathrm{H}), 7.84(\mathrm{~m}, 2 \mathrm{H})$, $9.69(\mathrm{~s}, 1 \mathrm{H})$.

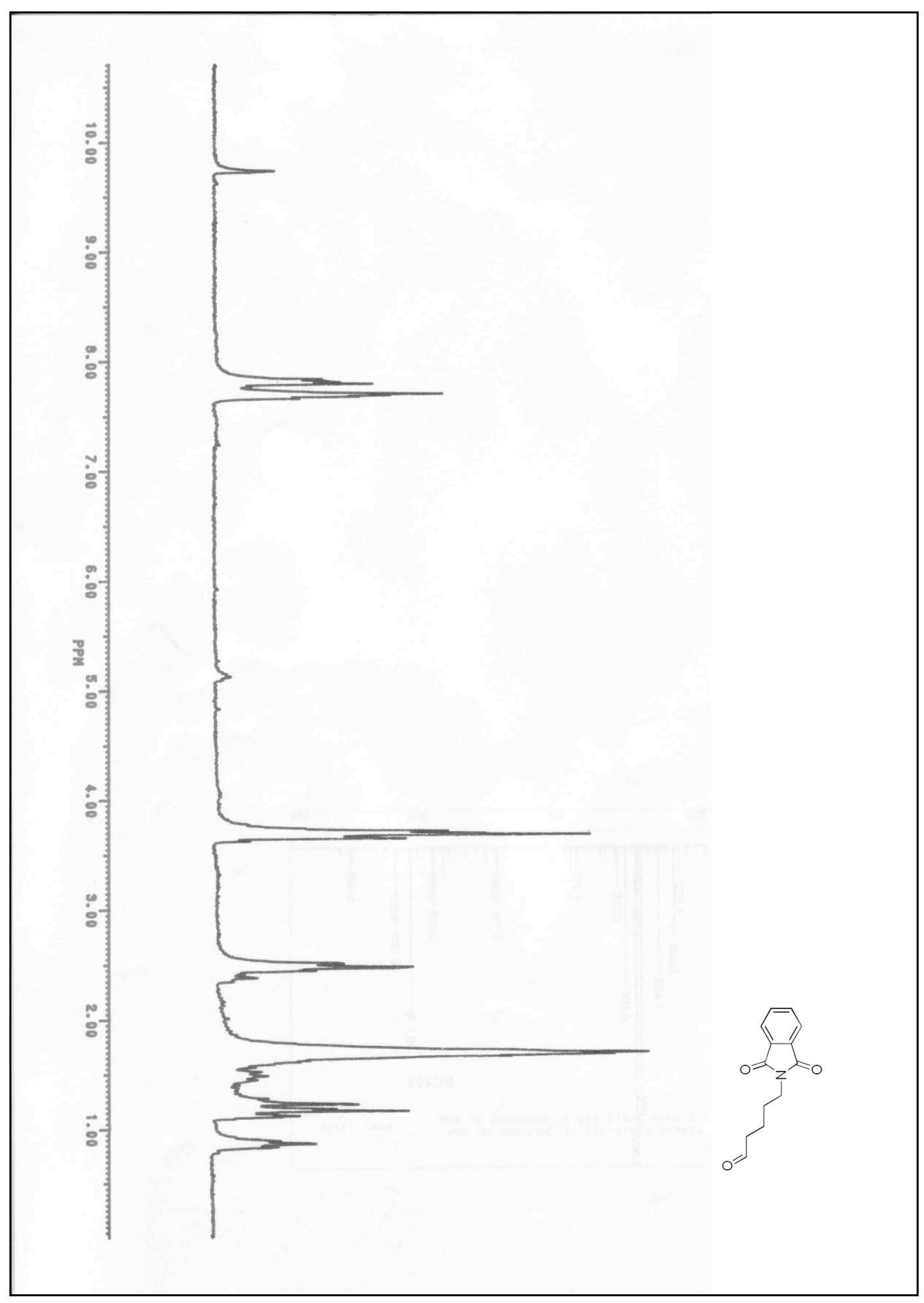

12 


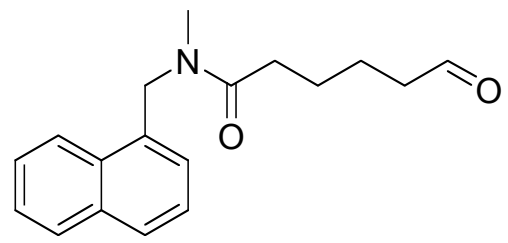

19

${ }^{1} \mathrm{H}$ NMR (200 MHz,CDCl $): 1.72(\mathrm{~m}, 4 \mathrm{H}), 2.34(\mathrm{~m}, 4 \mathrm{H}), 2.83(\mathrm{~s}, 3 \mathrm{H}), 4.99(\mathrm{~d}, J=8.5,2 \mathrm{H}), 7.33$ (m, 5H), $7.85(\mathrm{~m}, 1 \mathrm{H}), 8.01(\mathrm{~m}, 1 \mathrm{H}), 9.62(\mathrm{~s}, 1 \mathrm{H}), 9.71(\mathrm{~s}, 1 \mathrm{H}) .{ }^{13} \mathrm{C} \mathrm{NMR}\left(200 \mathrm{MHz}, \mathrm{CDCl}_{3}\right): 21.6$, $24.55,32.3,33.2$, 43.6, 48.5, 123.8, 125.1, 125.6, 125.91, 127.0, 128.2, 128.4, 131.7, 132.7, 133.8, 202.0. $\mathrm{m} / \mathrm{z}(\mathrm{ES}-\mathrm{MS})=306\left[\mathrm{M}^{+}+\mathrm{Na}\right]$ HRMS Calcd for $\mathrm{C}_{18} \mathrm{H}_{21} \mathrm{NO}_{2}$ Exact Mass: 283.1572. Found 283.1569 .

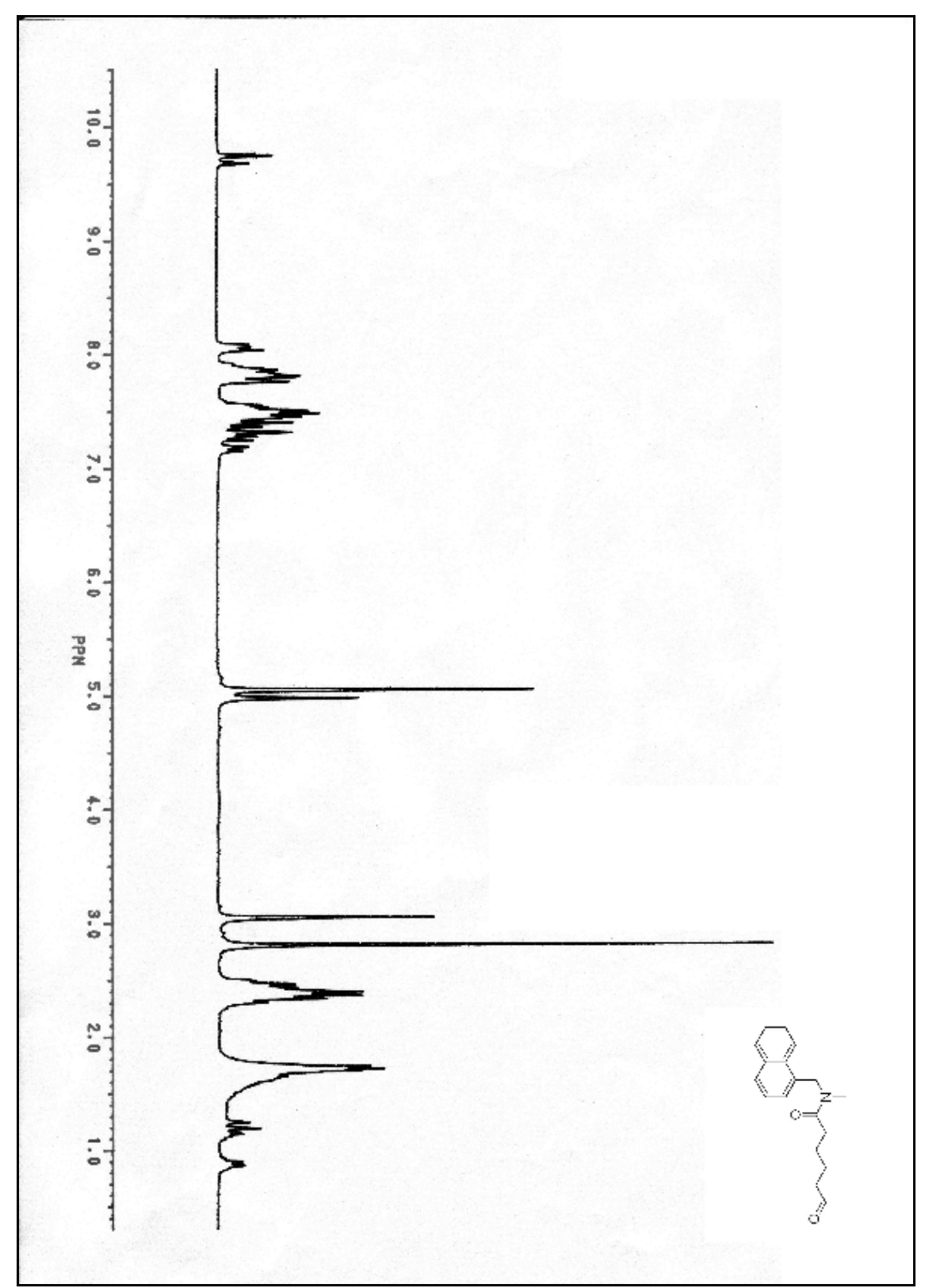




$$
1
$$




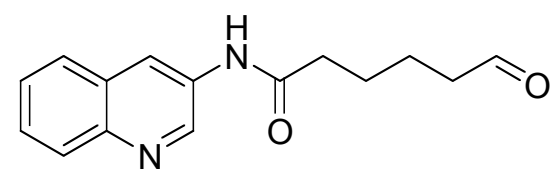

22

${ }^{1} \mathrm{H}$ NMR (200 MHz,CDCl $) 1.78(\mathrm{~m}, 4 \mathrm{H}), 2.47$ (m, 2H), 2.69, (m, 2H), 7.55 (m, 4H), 7.92 (m, 1H), 8.65 (m, 1H), $9.77(\mathrm{~s}, 1 \mathrm{H}) .{ }^{13} \mathrm{C}$ NMR (200 $\left.\mathrm{MHz} \mathrm{CDCl}_{3}\right)$ 22.15, 25.71, 37.24, 44.1, 126.01, 126.53, 128.15, 128.95, 131.97, 152.04, 201.71. $\mathrm{m} / z: 257[\mathrm{M}]^{+}, 279[\mathrm{M}+\mathrm{Na}]^{+}$. HRMS Calcd for $\mathrm{C}_{15} \mathrm{H}_{16} \mathrm{~N}_{2} \mathrm{O}_{2} ; 256,1212$. Found 256.1210.

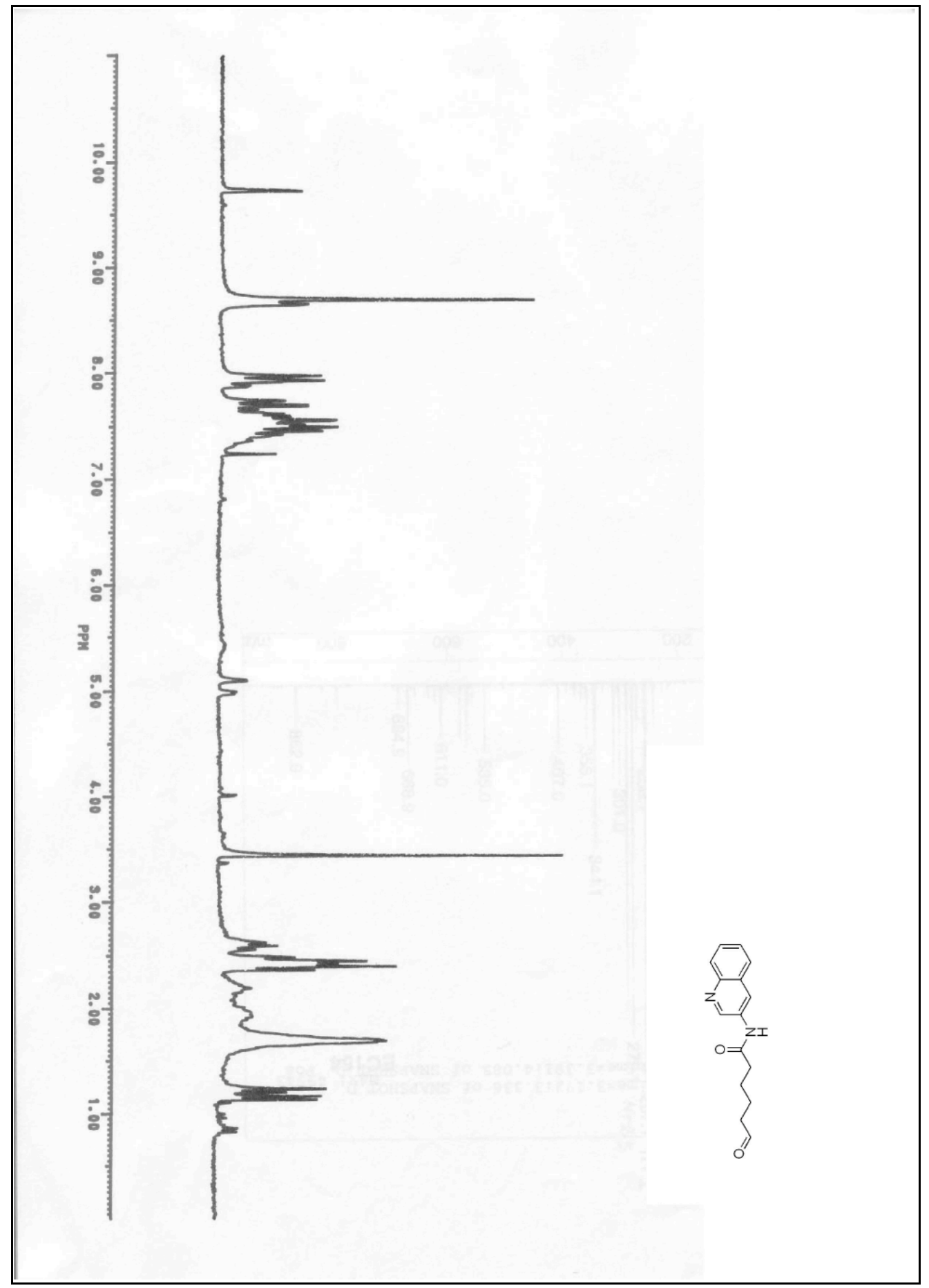





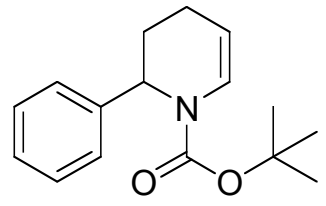

25

${ }^{1} \mathrm{H}$ NMR (200 MHz,CDCl $) 1.42(\mathrm{~s}, 9 \mathrm{H}), 1.71(\mathrm{~m}, 2 \mathrm{H}), 2.05(\mathrm{~m}, 2 \mathrm{H}), 4.86(\mathrm{~m}, 1 \mathrm{H}), 5.37(\mathrm{~m}, 1 \mathrm{H})$, $7.03(\mathrm{~m}, 1 \mathrm{H}), 7.17$ (m, 5H). ${ }^{13} \mathrm{C}$ NMR (200 $\left.\mathrm{MHz} \mathrm{CDCl}_{3}\right)$ 23.75, 29.15, 38.51, 57.82, 73.41, 108.67, 125.37, 126.53, 128.44, 136.96, 151.38. $\mathrm{m} / \mathrm{z}$ (ES-MS): $282[\mathrm{M}+\mathrm{Na}]^{+}$. HRMS Calcd for $\mathrm{C}_{16} \mathrm{H}_{21} \mathrm{NO}_{2}, 259,1572$. Found 259.1569.

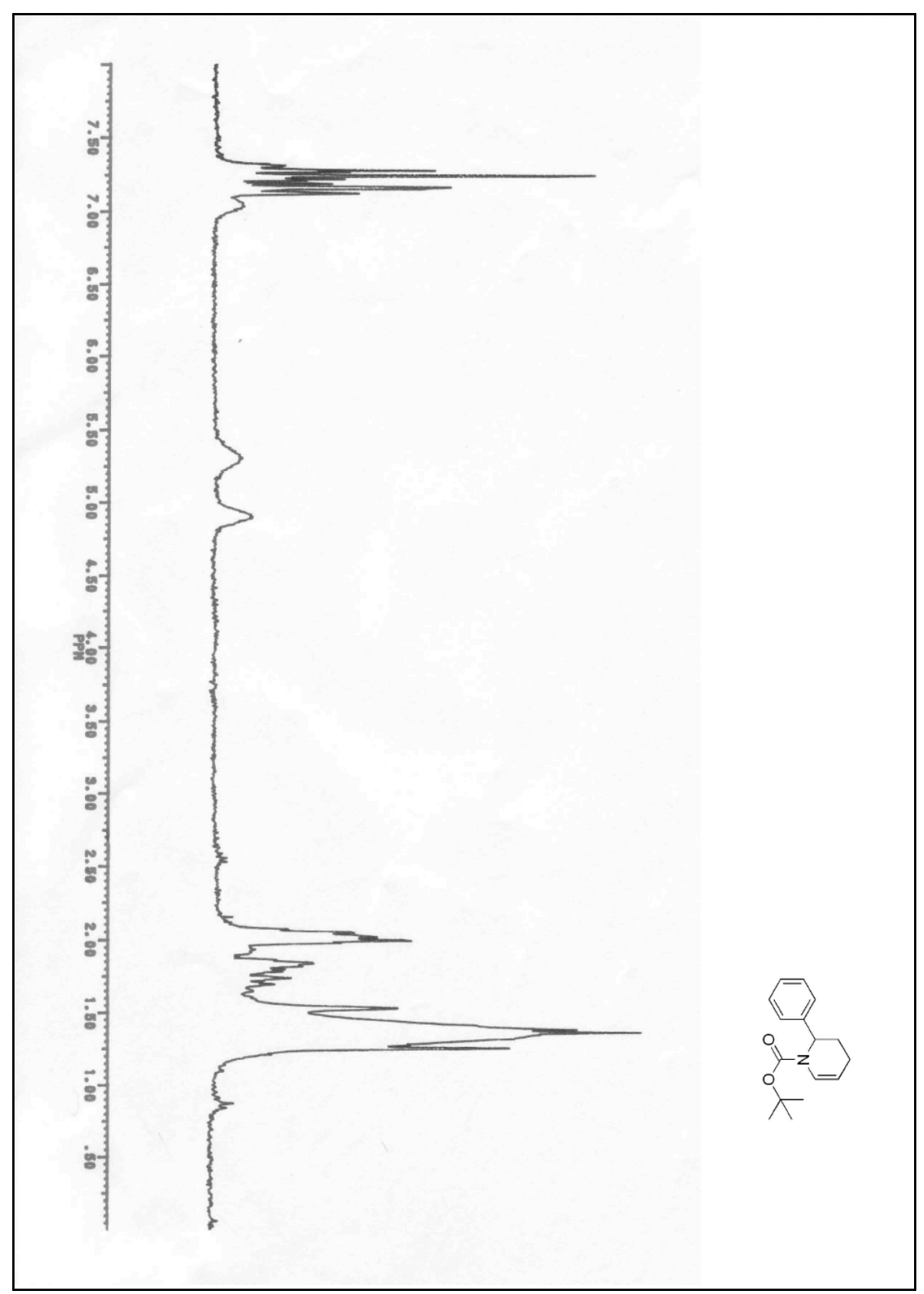




$$
\mid
$$




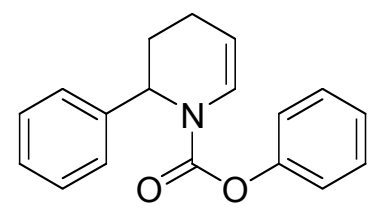

26

${ }^{1} \mathrm{H}$ NMR (200 MHz,CDCl 3$) 1.71(\mathrm{~m}, 2 \mathrm{H}), 2.15(\mathrm{~m}, 2 \mathrm{H}), 4.98(\mathrm{~m}, 1 \mathrm{H}), 5.35(\mathrm{~m}, 1 \mathrm{H}), 7.44(\mathrm{~m}$, $11 \mathrm{H}) .{ }^{13} \mathrm{C} \mathrm{NMR}\left(200 \mathrm{MHz}, \mathrm{CDCl}_{3}\right)$ 29.68, 39.40, 55.41, 195.10, 123.02, 125.49, 126.83, 128.39, 137.88, 152.99. $\mathrm{m} / z$ (ES-MS): $302[\mathrm{M}+\mathrm{Na}]^{+}$. HRMS Calcd for $\mathrm{C}_{18} \mathrm{H}_{17} \mathrm{NO}_{2}$ Exact Mass: 279.1259 Found 279.1256.

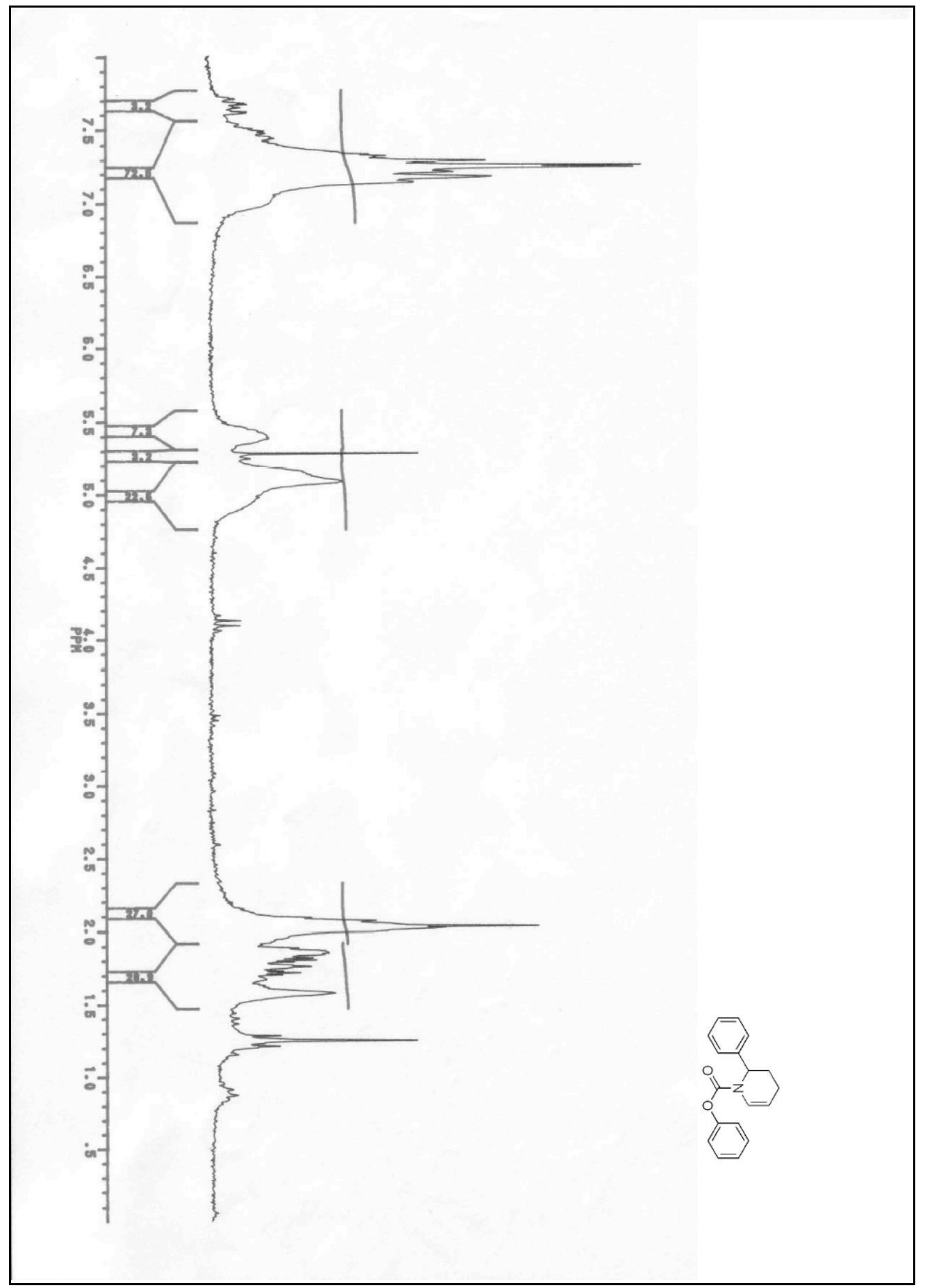




$$
\mid
$$




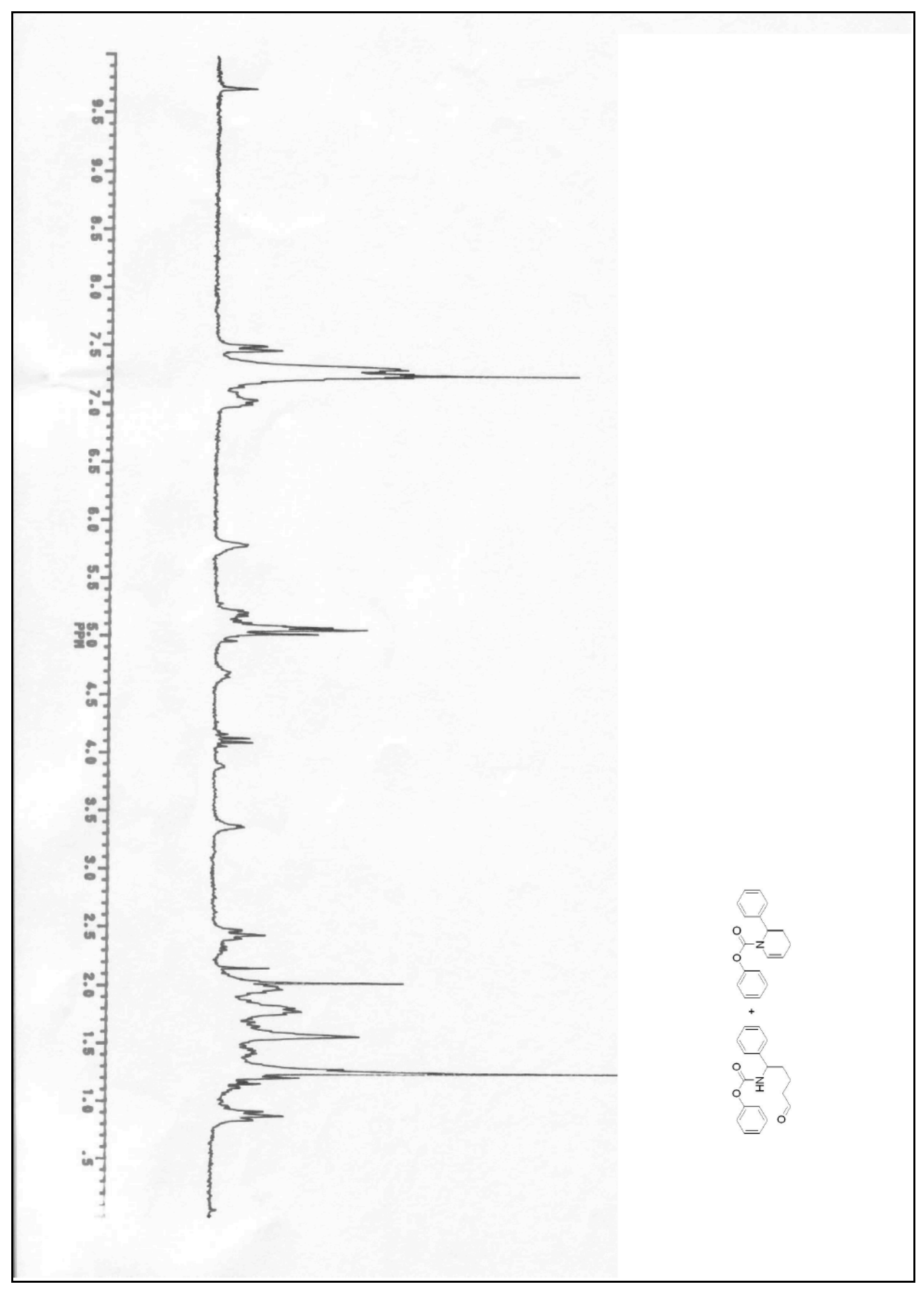

21 


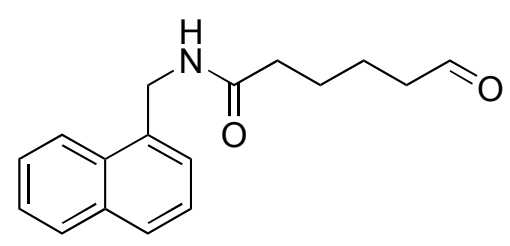

20

${ }^{1} \mathrm{H}$ NMR (200 MHz, $\left.\mathrm{CDCl}_{3}\right): 1.77(\mathrm{~m}, 4 \mathrm{H}), 2.30(\mathrm{~m}, 5 \mathrm{H}), 4.76(\mathrm{~d}, J=8.5,2 \mathrm{H}), 7.30(\mathrm{~m}, 5 \mathrm{H}), 7.85$ $(\mathrm{m}, 1 \mathrm{H}), 8.00(\mathrm{~m}, 1 \mathrm{H}), 9.62(\mathrm{~s}, 1 \mathrm{H}), 9.76(\mathrm{~s}, 1 \mathrm{H}) .{ }^{13} \mathrm{C} \mathrm{NMR}\left(200 \mathrm{MHz}, \mathrm{CDCl}_{3}\right): 21.8,24.5,34.3$, 33.9, 49.7, 123.9, 125.0, 125.6, 125.9, 127.0, 128.2, 128.3, 131.9, 133.0, 133.8, 200.7. m/z: ES/MS $292[\mathrm{M}+\mathrm{Na}]^{+}$. Anal. Calcd for $\mathrm{C}_{17} \mathrm{H}_{19} \mathrm{NO}_{2}: \mathrm{C}, 75.81 ; \mathrm{H}, 7.11 ; \mathrm{N}, 5.20$. Found: C, 75.89; H, 7.09; N, 5.19 .

1 Susan, K.; Cross, R. J.; Farrugia, L.J.; Nichols, D.A.; Perry, A. Eur. J. Inorg. Chem. 2002, 1, 141152.

2 Artaud, I.; Ben-Aziza, K.; Monsuy, D. J. Org. Chem. 1993, 58, 3373-3380.

3 Monfilier, E.; Tilloy, S.; Fremy, G.; Castanet, Y.; Mortreux, A. Tetrahedron Lett. 1995, 52, 94819484.

4 Saphier, S.; Hu, Y.; Sinha, S. C.; Houk, K. N.; Keinan, E. J. Am.Chem. Soc. 2005, 127, 132-145.

5 Douglass, F.T., Dawei, T. J. Org. Chem. 2002, 67, 1607-1612.

6 Trost, B.;M.; Kulawiec, R.J. J. Am. Chem. Soc. 1993, 115, 2027-2036.

7 Delwin, S.; Frasen, S.A.; Ni, L.-M.; Kam, C.-M.; Winkler, U. et al. J. Med. Chem. 1998, 41, 2289-2301. 